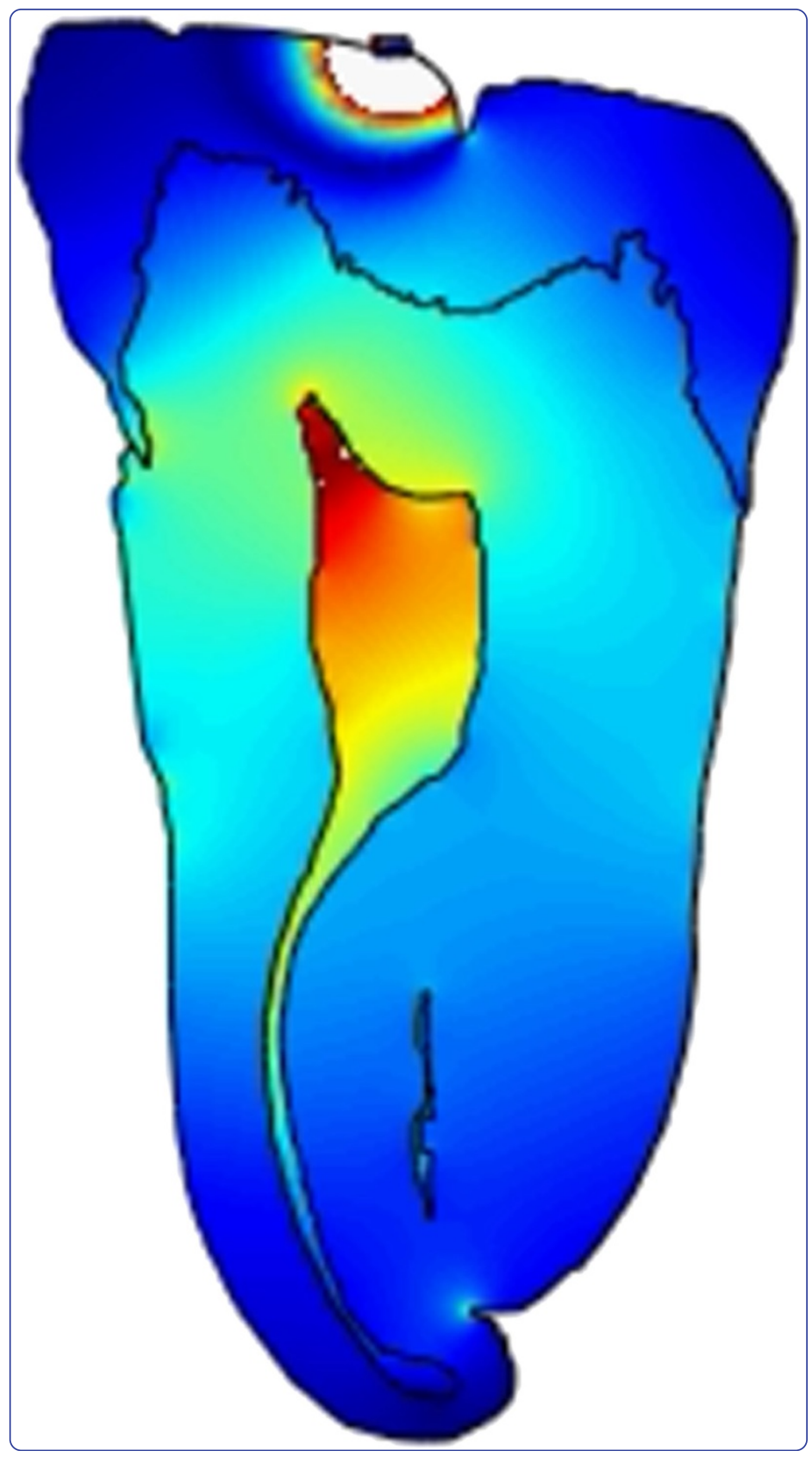

\title{
Biophysical characterization of low-frequency ultrasound interaction with dental pulp stem cells
}

Ghorayeb et al. 


\title{
Biophysical characterization of low-frequency ultrasound interaction with dental pulp stem cells
}

\author{
Sleiman R Ghorayeb ${ }^{1,2^{*}}$, Upen S Patel ${ }^{3}$, A Damien Walmsley ${ }^{3}$ and Ben A Scheven ${ }^{3}$
}

\begin{abstract}
Background: Low-intensity ultrasound is considered an effective non-invasive therapy to stimulate hard tissue repair, in particular to accelerate delayed non-union bone fracture healing. More recently, ultrasound has been proposed as a therapeutic tool to repair and regenerate dental tissues. Our recent work suggested that low-frequency kilohertz-range ultrasound is able to interact with dental pulp cells which could have potential to stimulate dentine reparative processes and hence promote the viability and longevity of teeth.

Methods: In this study, the biophysical characteristics of low-frequency ultrasound transmission through teeth towards the dental pulp were explored. We conducted cell culture studies using an odontoblast-like/dental pulp cell line, MDPC-23. Half of the samples underwent ultrasound exposure while the other half underwent 'sham treatment' where the transducer was submerged into the medium but no ultrasound was generated. Ultrasound was applied directly to the cell cultures using a therapeutic ultrasound device at a frequency of $45 \mathrm{kHz}$ with intensity settings of 10,25 and $75 \mathrm{~mW} / \mathrm{cm}^{2}$ for $5 \mathrm{~min}$. Following ultrasound treatment, the odontoblast-like cells were detached from the culture using a $0.25 \%$ Trypsin/EDTA solution, and viable cell numbers were counted. Two-dimensional tooth models based on $\mu-C T 2 D$ images of the teeth were analyzed using COMSOL as the finite element analysis platform. This was used to confirm experimental results and to demonstrate the potential theory that with the correct combination of frequency and intensity, a tooth can be repaired using small doses of ultrasound. Frequencies in the $30 \mathrm{kHz}-1 \mathrm{MHz}$ range were analyzed. For each frequency, pressure/intensity plots provided information on how the intensity changes at each point throughout the propagation path. Spatial peak temporal average (SPTA) intensity was calculated and related to existing optimal spatial average temporal average (SATA) intensity deemed effective for cell proliferation during tooth repair.
\end{abstract}

Results: The results demonstrate that odontoblast MDPC-23 cell numbers were significantly increased following three consecutive ultrasound treatments over a 7-day culture period as compared with sham controls underscoring the anabolic effects of ultrasound on these cells. Data show a distinct increase in cell number compared to the sham data after ultrasound treatment for intensities of 10 and $25 \mathrm{~mW} / \mathrm{cm}^{2}$ ( $p<0.05$ and $p<0.01$, respectively). Using finite element analysis, we demonstrated that ultrasound does indeed propagate through the mineralized layers of the teeth and into the pulp chamber where it forms a 'therapeutic' force field to interact with the living dental pulp cells. This allowed us to observe the pressure/intensity of the wave as it propagates throughout the tooth. A selection of time-dependent snapshots of the pressure/intensity reveal that the lower frequency waves propagate to the pulp and remain within the chamber for a while, which is ideal for cell excitation. Input frequencies and pressures of $30 \mathrm{kHz}$ (70 Pa) and $45 \mathrm{kHz}\left(31 \mathrm{kPa}\right.$ ), respectively, with an average SPTA of up to $120 \mathrm{~mW} / \mathrm{cm}^{2}$ in the pulp seem to be optimal and agree with the SATA intensities reported experimentally.

(Continued on next page)

\footnotetext{
* Correspondence: Sleiman.R.Ghorayeb@hofstra.ed

${ }^{1}$ School of Engineering and Applied Sciences, Ultrasound Research

Laboratory, Hofstra University, 104 Weed Hall, Hempstead, NY 11549, USA

${ }^{2}$ Orthopaedics Research Laboratory, FIMR, North Shore Hospital, Manhassett,

NY 11030, USA

Full list of author information is available at the end of the article
}

\section{Biomed Central}

(c) 2013 Ghorayeb et al.; licensee BioMed Central Ltd. This is an Open Access article distributed under the terms of the Creative Commons Attribution License (http://creativecommons.org/licenses/by/2.0), which permits unrestricted use, distribution, and reproduction in any medium, provided the original work is properly cited. 
(Continued from previous page)

Conclusions: Our data suggest that ultrasound can be harnessed to propagate to the dental pulp region where it can interact with the living cells to promote dentine repair. Further research is required to analyze the precise physical and biological interactions of low-frequency ultrasound with the dental pulp to develop a novel non-invasive tool for dental tissue regeneration.

Keywords: Therapeutic ultrasound, Kilohertz-range ultrasound, Dental tissues, Tooth, Dental pulp, Tissue repair and regeneration, Dentine repair, Finite element modelling

\section{Background}

Ultrasound has various industrial and medical applications. In medical imaging, it is recognized as an important and useful clinical tool in (prenatal) screening, diagnostics and surgery. In dentistry, ultrasound use is mostly limited to oral surface cleaning (i.e. removal of plaque and calculus) or root canal treatment [1]. Studies have also evaluated the use of high-frequency imaging ultrasound for dental diagnostic purposes as reported by Ghorayeb et al. [2,3].

Therapeutic ultrasound for healing and repair of tissues following injury or disease is increasingly gaining interest in the scientific and clinical community. In particular, the notion that low-intensity pulsed ultrasound is an effective tool to accelerate bone fracture healing highlights the exciting potential of ultrasound in hard tissue repair and engineering [4-6]. This paper addresses the question as to whether ultrasound can be used as a non-invasive biomechanical therapy to promote dental health and tissue repair. Oral health is essential for human health and well being, whilst dental disease affects the quality of life for individuals worldwide imposing an immense burden on healthcare systems as reported by the World Health Organization [7]. Despite advances in restorative materials, traditional dental treatments using filling materials are relatively inefficient with approximately $50 \%$ of cases requiring revision within 5-10 years after treatment [8].

Teeth are living biomechanical tissues consisting of mineralized dentine which is covered by highly mineralized and non-vital enamel. The dentine has a tubular structure containing fluid and cell processes from odontoblasts that are located around the periphery of the viable pulp chamber within the core of teeth. Odontoblasts are viable and active throughout life and are responsible for dentine production. Following mild tooth decay or injury, the activity of existing odontoblasts can be upregulated to produce reactionary dentine involving formation and repair of extracellular mineralized (inter- and intratubular) dentine matrix that serves to seal off and protect the viable dental pulp core. However, in the event of a major obnoxious damage resulting in (localized) death of odontoblasts, progenitor or stem cells residing in the dental pulp can be activated to differentiate to new odontoblasts which in turn produce new reparative dentine $[9,10]$.

Accumulating evidence from pre-clinical studies has indicated that low-intensity pulsed ultrasound is capable of directly stimulating the cartilage and bone cells to accelerate bone repair and regeneration [5,6,11-13]. Our previous work demonstrated the significant potential of ultrasound to stimulate dental cells $[10,14,15]$.

Finite element (FE) methods are widely used in the understanding of physical and mechanical behaviour in various structures. FE has been used to solve equations which govern ultrasonic wave propagation in teeth [16-18]. Ghorayeb and colleagues showed that inflammation of the pulp could be modelled by density changes in the pulpal area [19]. The particular novelty of this project proposal is the analysis of 'therapeutic' lowfrequency ultrasound propagation and its interaction with odontoblasts and the dental pulp. Apart from the complex multilayered and anisotropic tissue structure, the challenge herein is the relative low frequency (up to $1 \mathrm{MHz}$ ) and thus long wave range of therapeutic lowintensity (kHz-range) ultrasound. Recently, 2D finite difference modelling was used to estimate low-frequency ultrasound propagation in bone-like samples [20]. In addition, modelling techniques have been shown to be effective for simulating ultrasound propagation and interaction with complex structures and materials, including bone and periodontal ligament [21-23]. These approaches allow high-fidelity simulations to be benchmarked directly to biological experiments and then used systematically to understand in detail the internal interactions of ultrasound with intricate tissue structures. In order to better understand low-frequency and low-intensity ultrasound propagation in teeth and its interaction with the vital dental pulp core, this study is aimed to generate and analyze 2D models of tooth using micro-computed tomography $(\mu-\mathrm{CT})$ and finite element modelling. In addition, in order to relate the computational modelling with biological effect, we investigated the direct effects of different intensities of low-frequency ultrasound on dental pulp cells using an odontoblastlike pulpal cell line. 


\section{Methods}

Scheven et al. $[14,15]$ investigated the effects of lowfrequency (kHz-range) ultrasound, generally used in dentistry for dental scaling, on odontoblast-like cells. These studies have shown that a single exposure of odontoblast-like cell lines to low-frequency ultrasound resulted in distinct effects on cell vitality and cell behaviour. Ultrasound was able to stimulate the expression of genes and production of growth factors such as transforming growth factor $\beta 1$ (TGF $\beta 1$ ) and vascular endothelial growth factor (VEGF) believed to be important for odontoblast activity and dentine repair. These promising data highlight the significant potential for the exploitation of ultrasound in novel dental regenerative therapies. This notion is supported by previous observations by Olgart et al. [24] who described that treatment of teeth with low-frequency ultrasound stimulated pulpal blood flow. Taken together, we have postulated that exposure of teeth to ultrasound may trigger cellular responses in the dentine-pulp complex, thereby possibly stimulating odontoblast activity and/or activating pulp mesenchymal stem cells inducing tooth repair $[10,15]$. Little is known, however, about the underlying biophysical mechanisms of action of ultrasound in hard tissues. Moreover, the precise relationship of ultrasound intensity and frequency with biological effect is unclear.

All studies were approved by and in compliance with the guidelines set by the institutional review board committee at the School of Dentistry, College of Medical and Dental Sciences, University of Birmingham (Birmingham, UK) and at Hofstra University (Hempstead, NY, USA).

\section{Finite element modelling}

This portion of the study addressed the characteristics of ultrasonic waves travelling through the different layers of mineralized dental tissues (enamel, dentine) and their interaction with the dentine-pulp complex using computational simulation modelling. Finite element was used to explore the potential theory that with the correct combination of frequency and intensity, a tooth can be repaired using small doses of ultrasound. In addition, the purposes of this study are (a) to accurately model the waves propagating in the teeth, (b) to observe safety issues by keeping track of the spatial peak temporal average (SPTA) intensity and (c) to relate this figure to existing optimal spatial average temporal average (SATA) intensity deemed effective for cell proliferation during tooth repair. It is envisaged that ultimately, these computational analyses will be related to biological effects and would facilitate the development of a dental therapeutic ultrasound device. The SPTA intensity $\left(I_{\text {spta }}\right)$ was calculated using the following relationship [17]:

$$
I_{\mathrm{spta}}=\frac{1}{T_{\mathrm{prf}}} \int \frac{p^{2}(0, F, t)}{\rho c_{o}}
$$

where $T_{\text {prf }}$ is the pulse repetition period ( $=1 \mathrm{~ms}$ ), $\rho$ is the density, $c_{\mathrm{o}}$ is the sound velocity in the tooth layers (Table 1) and $p(0, F, t)$ is the ultrasonic pressure in the tooth calculated using the following:

$$
p(0, F, t)=\rho h_{o} \frac{\partial v(t)}{\partial t}
$$

where $v(t)$ is the velocity at the transducer-medium interface, and $h_{\mathrm{o}}$ is the depth at which the spatial peak intensity across the entire radiated ultrasonic beam is being measured. For a given focal length $F$ and transducer disc radius $a, h_{\mathrm{o}}$ can be determined from the following:

$$
h_{o}=F-\sqrt{\left(F^{2}-a^{2}\right)}
$$

The intent of this simulation was to see what combinations of frequencies and input pressures would yield a range of SPTA intensities between 30 and $120 \mathrm{~mW} / \mathrm{cm}^{2}$ in the pulp region and then confirm that this level is maintained throughout the entire tooth in accordance with FDA regulations. Furthermore, this range (30-120 $\mathrm{mW} / \mathrm{cm}^{2}$ ) was adopted as the absolute limit threshold at low ultrasonic frequencies that would stimulate bone repair and may also apply to tooth regeneration as determined using dental scalers $[10,14,15]$.

Two-dimensional tooth models were analyzed using COMSOL (COMSOL, Inc., Burlington, MA, USA). Figure 1 shows typical $\mu$-CT 2D images of the teeth. These were loaded into the acoustics module of COMSOL as the platform to be discretized and analyzed.

The input pressure pulse used is $\cos (2 \pi f t) \times$ $\left(1-\cos \left(2 \pi f \frac{t}{3}\right)\right)$, where $f$ is the acoustic frequency in Hertz, and $t$ is time in seconds. Frequencies in the $30 \mathrm{kHz}-1 \mathrm{MHz}$ range were analyzed. For each frequency, the pressure/intensity plots provided information on how the intensity changes at each point throughout the propagation path.

\section{Cell cultures and ultrasound application}

MDPC-23 is a proliferating cell line derived from fetal murine dental papilla expressing a range of odontoblast-

\begin{tabular}{|c|c|c|}
\hline Material & Density $\left(\mathrm{kg} / \mathrm{m}^{3}\right)$ & Speed of sound $(\mathrm{m} / \mathrm{s})$ \\
\hline Enamel & 3,000 & 6,250 \\
\hline Dentin & 2,000 & 3,800 \\
\hline Pulp & 1,000 & 1,570 \\
\hline
\end{tabular}
like characteristics $[14,15]$. The MDPC-23 cells were

Table 1 Densities and speed of sound in each tooth layer 


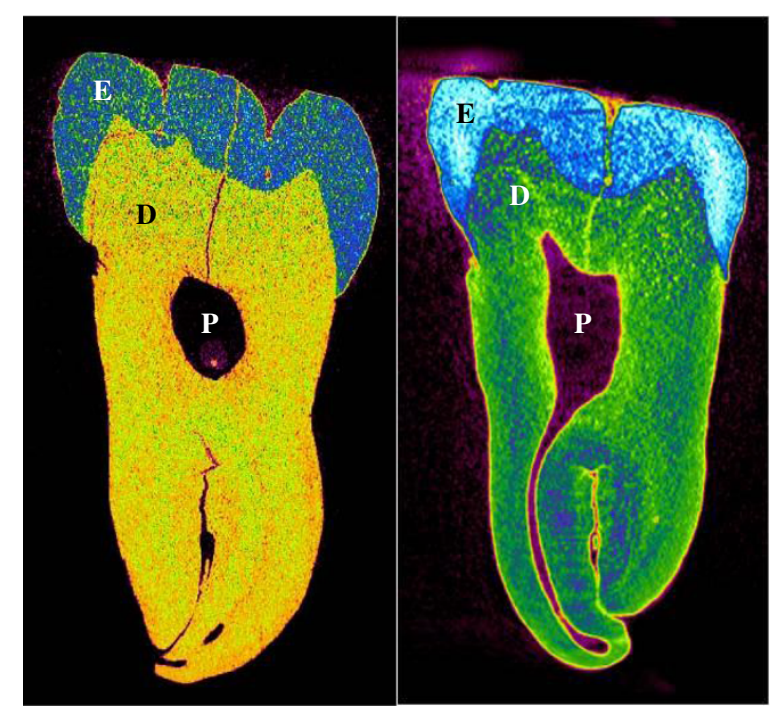

Figure $1 \mu$-CT scans of two different teeth showing all three major layers including the pulp cavity. $E$, enamel; $D$, dentine; $P$, pulp.

cultured as an adherent monolayer in T75 flasks containing Dulbecco's modified Eagle Medium (DMEM) supplemented with $10 \%$ fetal bovine serum, $1 \%$ penicillin/streptomycin (Sigma-Aldrich ${ }^{\circ}$, Dorset, England, UK) and $200 \mathrm{mM}$ glutamine (GlutaMAX ${ }^{\mathrm{m}}$, Gibco ${ }^{\circ}$, Invitrogen $^{\mathrm{Tm}}$, Sigma-Aldrich ${ }^{\circ}$ ) in a humidified incubator with $5 \%$ carbon dioxide in air at $37^{\circ} \mathrm{C}$. Near confluent cultures were detached from the culture plastic using Trypsin/EDTA (GIBCO, Paisley, UK) treatment, resuspended into a homogenous single cell suspension and seeded in three 6-well plates (Costar ${ }^{\circ}$ tissue-culture treated, Corning ${ }^{\circ}$, Corning, NY, USA). On day zero, 50,000 cells were seeded in each well of the three 6-well plates and subsequently formed an adherent monolayer. The culture medium was replenished on days 1, 3, 5 and 7 with ultrasound treatment on days 2, 4 and 6. Each plate had three wells exposed to ultrasound and three control group that underwent 'sham treatment' where the transducer was submerged into the medium but no ultrasound was generated $\left(0 \mathrm{~mW} / \mathrm{cm}^{2}\right)$. Ultrasound was applied directly to the cell cultures using a therapeutic ultrasound device (Duo Son, SRA Developments, Devon, UK) at a frequency of $45 \mathrm{kHz}$ with intensity settings of 10,25 and $75 \mathrm{~mW} / \mathrm{cm}^{2}$ (SATA) for $5 \mathrm{~min}$. On day 8 of culture, following ultrasound treatment, the odontoblastlike cells were detached from the culture using a $0.25 \%$ Trypsin/EDTA solution (Sigma-Aldrich ${ }^{\circ}$ ), and viable cell numbers were counted using a haemocytometer and trypan blue staining. Ultrasound intensity was calibrated using a hydrophone force field analyzer (SRA Developments, Devon, UK). Experimental temperature monitored using a digital thermometer (Iso-Tech IDM 207,
Northants, UK) with a 'K-type' wire showed that the temperature of the culture medium only marginally increased by approximately $2-3^{\circ} \mathrm{C}$ after $30 \mathrm{~min}$ of ultrasound application indicating that thermal stress was negligible in the current experimental set-up.

\section{Results and discussion}

\section{FE simulation results}

Figure 2 shows the discretized tooth geometry.

Surface plots were superimposed over contour plots, and animations of these plots were created. This allowed us to see the pressure/intensity throughout the tooth while watching the wave propagate. The following results shown in Figures 3, 4, 5 and 6 (Additional files 1, 2,3 and 4, respectively) exemplify a selection of timedependent snapshots taken for frequencies in the 30 $\mathrm{kHz}-1 \mathrm{MHz}$ range. For each frequency, the pressure/intensity plots are shown, providing information on how

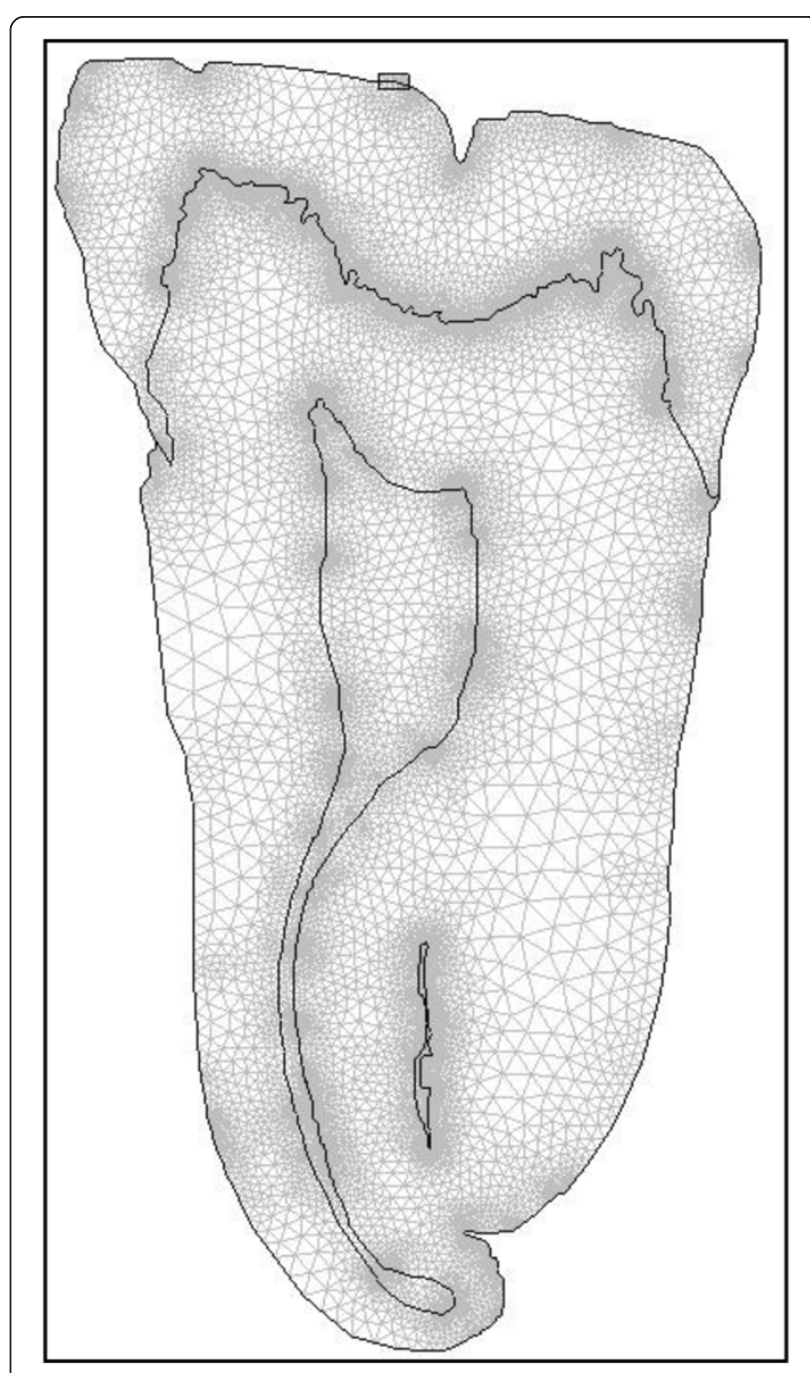

Figure 2 Discretization of one of the CT tooth scans. 
(a)

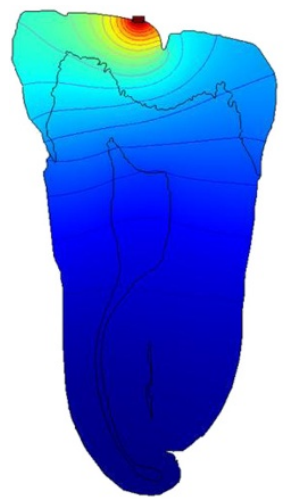

(b)

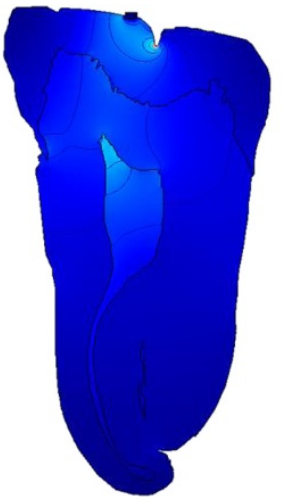

(c)

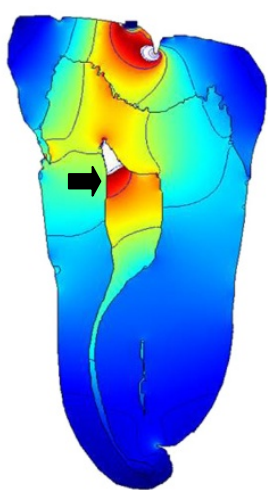

Figure 3 Snapshots of pressure and intensity plots of $(30 \mathrm{kHz}, 70 \mathrm{~Pa})$ ultrasonic wave throughout the tooth. (a) Pressure at $t=1.5 \mu \mathrm{S}$, (b) intensity at $t=0.5 \mu \mathrm{s}$ and $(\mathbf{c})$ intensity at $t=3 \mu \mathrm{s}$. It shows high-intensity concentration in pulp (arrow) (Additional file 1).

the intensity changes at each point throughout the propagation path.

The data as presented in Table 2 indicate the 'best' combinations of frequencies and input pressures that are within FDA regulations for ultrasonic exposure limits in the pulp. This means that using one of these combinations guarantees that the ultrasound intensity in the pulp will not exceed the maximum SPTA intensity of $120 \mathrm{~mW} / \mathrm{cm}^{2}$ calculated using Equation 1.

Interestingly, when the pressures were plotted against their respective frequencies, there seemed to be no direct correlation that is valid for predicting more values, as seen in Figure 7.
Examining the results, it appears that the lower frequency waves propagate to the pulp and remain within the chamber for a while. This is optimal if considering the cells we want to excite are located there. The higher frequencies, due to the smaller wavelength, are more affected by imperfections in the boundaries, as reflected by the higher intensity surface waves that are distributed throughout the periphery of the tooth. Considering all of the results shown in Figures 3, 4, 5 and 6 (Additional files $1,2,3$ and 4 , respectively), the optimal input frequencies and pressures are the $30-\mathrm{kHz}(70 \mathrm{~Pa})$ and the $45-\mathrm{kHz}(31 \mathrm{kPa})$ cases, respectively, as they generate an average of $120 \mathrm{~mW} / \mathrm{cm}^{2}$ in the pulp. It is important (a)

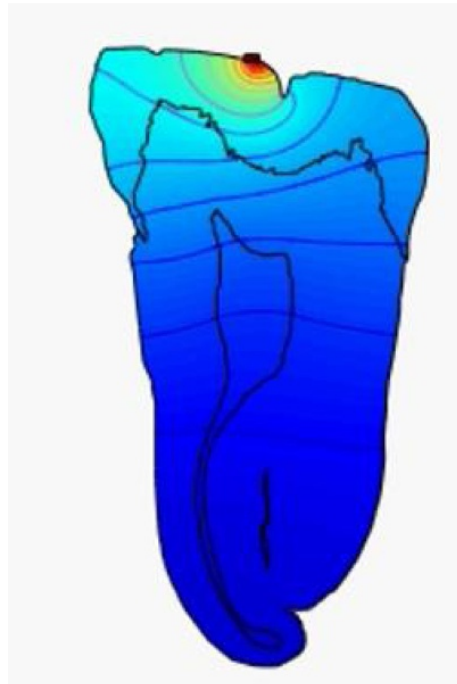

(b)

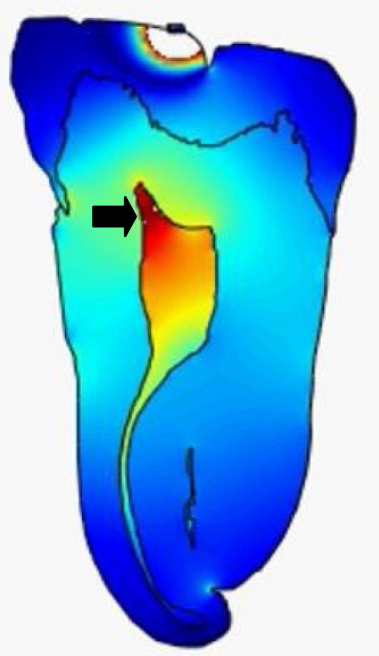

Figure 4 Snapshots of pressure and intensity plots of $(\mathbf{4 5 ~} \mathbf{k H z}, 31 \mathbf{k P a})$ ultrasonic wave throughout the tooth. (a) Pressure at $t=1 \mu \mathrm{s}$ and (b) intensity at $t=3.35 \mu \mathrm{s}$. It shows high-intensity concentration in pulp (arrow) (Additional file 2). 
(a)

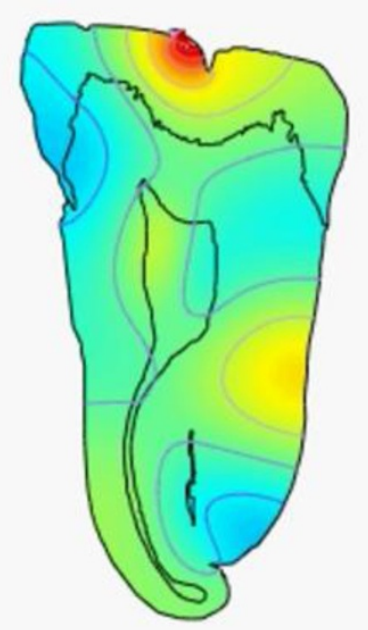

(b)

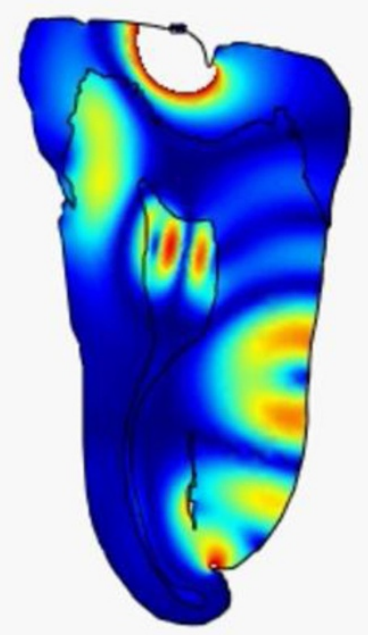

Figure 5 Snapshots of pressure and intensity plots of $(500 \mathbf{~ k H z}, 200 \mathrm{kPa})$ ultrasonic wave throughout the tooth. (a) Pressure at $t=1 \mu \mathrm{s}$ and (b) intensity at $t=3 \mu$ s (Additional file 3).

to note that these frequencies correspond to standard dental scalers such as those of the Cavitron ${ }^{\circ}$ scaler (DENTSPLY Professional, York, PA, USA) commonly used during dental hygiene and that Scheven et al. [14] and Olgart et al. [24] referred to in their studies. These cases can be seen as peak intensities (shown in red levels) in Figures 3 and 4 (Additional files 1 and 2). In these plots, any intensity over the limit of $120 \mathrm{~mW} / \mathrm{cm}^{2}$ would not be displayed on this greyscale. This means that the combination of frequency and pressure in these figures is FDA safe. Also, the input pressure is considerably lower than all other frequencies tested, making it even more attractive and efficient. However, it should be emphasized that these results refer to the safe upper limits of the therapeutic dental application theories, but that further detailed studies are needed to clarify and (a)

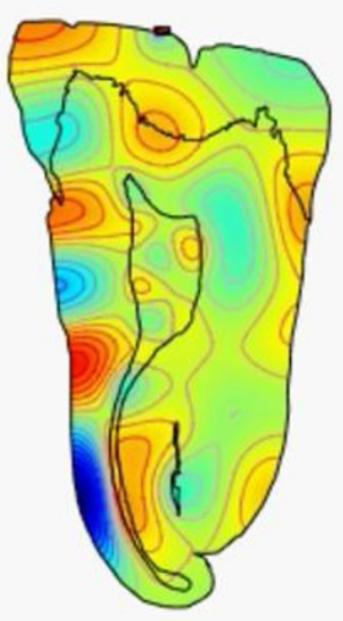

(b)

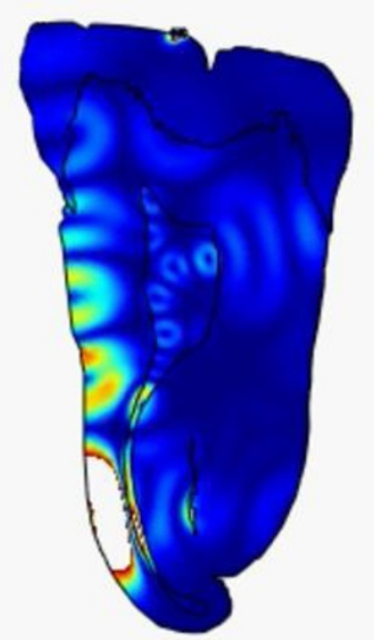

Figure 6 Snapshots of pressure and intensity plots of $(1 \mathrm{MHz}, 19 \mathrm{kPa})$ ultrasonic wave throughout the tooth. (a) Pressure at $t=1.5 \mu \mathrm{S}$ and (b) intensity at $t=1.5 \mu$ s (Additional file 4). 
Table 2 Upper limit for input pressures at the given frequencies

\begin{tabular}{lc}
\hline Frequency (Hz) & Input pressure (Pa) \\
\hline $30 k$ & 70 \\
$45 k$ & $43.5 k$ \\
$50 k$ & $127 k$ \\
$55 k$ & $85.5 k$ \\
$60 k$ & $109 k$ \\
$65 k$ & $149 k$ \\
$70 k$ & $139 k$ \\
$75 k$ & $340 k$ \\
$80 k$ & $200 k$ \\
$85 k$ & $400 k$ \\
$90 k$ & $210 k$ \\
$95 k$ & $231 k$ \\
$100 k$ & $170 k$ \\
$125 k$ & $59 k$ \\
$150 k$ & $162 k$ \\
$175 k$ & $214 k$ \\
$200 k$ & $135 k$ \\
$225 k$ & $80 k$ \\
$250 k$ & $221 k$ \\
$275 k$ & $149 k$ \\
$300 k$ & $300 k$ \\
$500 k$ & $90.1 k$ \\
$600 k$ & $25.65 k$ \\
$700 k$ & $331.6 k$ \\
$800 k$ & $84.88 k$ \\
$900 k$ & $15 k$ \\
$1 M$ & $55.7 k$ \\
\hline
\end{tabular}

thus confirm whether these regimens will provoke the specialization of dental pulp stem cells into odontoblastlike cells and therefore tooth regeneration.

\section{Low-frequency ultrasound stimulates MDPC-23 cell proliferation}

Further to the above-described simulations, the next experiments were conducted to investigate the biological effects of low-frequency (kHz-range) ultrasound on odontoblast-like cells and whether these effects are dose-dependent using a calibrated therapeutic ultrasound device. The results demonstrate that odontoblastlike MDPC-23 cell numbers were significantly increased following three consecutive ultrasound treatments over a 7-day culture period as compared with sham controls. The graph shown in Figure 8 is a compilation of three data sets of three experiments, using three replicates for each experiment. The data show the percentage change in cell number compared to the sham data after ultrasound treatment for each ultrasound intensity $(10,25$ and $\left.75 \mathrm{~mW} / \mathrm{cm}^{2}\right)$. The error bars represent standard deviation values $(p<0.05$ and $p<0.01)$ versus control values.

These findings indicate that ultrasound promoted cell proliferation at low-intensity therapeutic intensities. Ultrasound stimulation appeared to show a dose-dependent relationship with the greatest effect at intensities of 10 and $25 \mathrm{~mW} / \mathrm{cm}^{2}$. Interestingly, these values correspond to the intensities generally used in low-intensity pulsed ultrasound treatment for bone fracture healing $(25-30 \mathrm{~mW} /$ $\left.\mathrm{cm}^{2}\right)[5,6,25]$, although lower doses may be equally, if not, more effective in obtaining therapeutic effect. These findings also imply that biophysical stimulation by ultrasound may involve a threshold dose similar to the mechanostat threshold concept surrounding the theory of mechanical loading of bones [26,27]. However, there also seems an upper threshold where too high intensities may

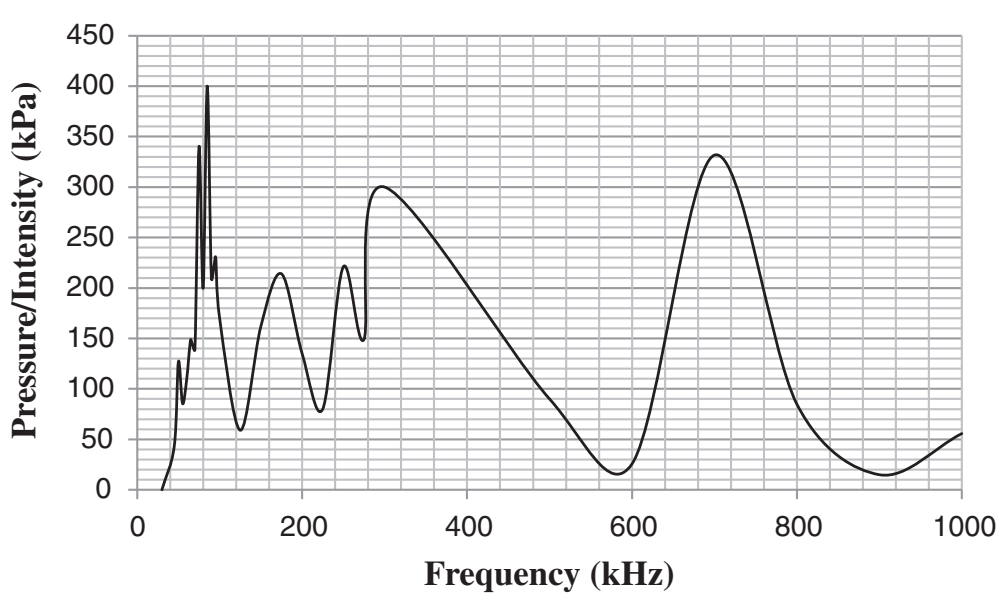

Figure 7 Plot showing relationship between ultrasonic wave pressure/intensity (in kPa) and frequency (in kHz). 


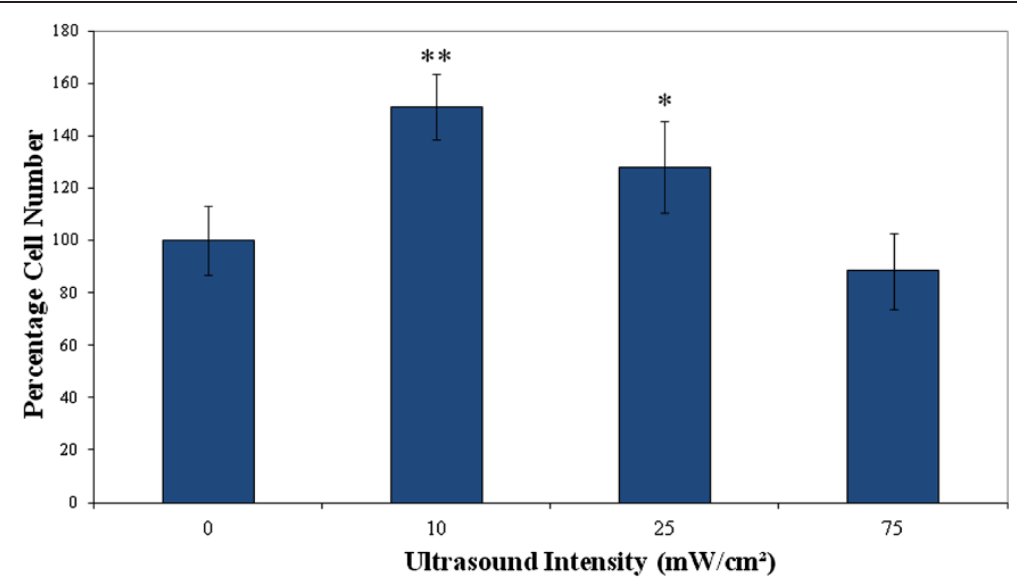

Figure 8 MDPC-23 odontoblast-like percent change in cell numbers after treatment with low-frequency $45-\mathrm{kHz}$ ultrasound. The data are mean of three experiments using three replicates for each experiment. The graph shows the percentage of cell number compared to the control group. The error bars shown represent standard deviation values versus control values ( $\left.p<0.05,{ }^{* *} p<0.01\right)$.

negate any positive biological/anabolic effect. These data linked with the computational modelling results shown earlier suggest that for safe and effective therapeutic use of dental ultrasound treatment, a relatively low-intensity application may deliver an efficient biological effect [28]. Our current findings were obtained from a 7-day culture experiment involving several consecutive ultrasound treatments.

Another point of clarification worth mentioning is that the SPTA and the SATA are related through the beam uniformity ratio (BUR). BUR is a quantitative indication of ultrasonic beam uniformity across the face of the transducer. A perfect ideal case is when BUR $=1$. However, as uniformity worsens, BUR increases. The transducer used in our therapeutic device (Duo Son, SRA Developments, Ashburton South Devon, UK) produces a non-uniform diverging beam with an effective beam radiating area of $16.3 \mathrm{~cm}^{2}$ and a BUR $\cong 6$. Spatial peak (SP) and spatial average (SA) figures are related by the following:

$$
\mathrm{SP}=(\mathrm{SA})(\mathrm{BUR})
$$

Therefore, our ideal SATA figures of 10 and $25 \mathrm{~mW} /$ $\mathrm{cm}^{2}$ obtained in the experimental results convert to SPTA values of 60 and $150 \mathrm{~mW} / \mathrm{cm}^{2}$, respectively, which overlap the range of SPTA intensity levels of 30-120 $\mathrm{mW} / \mathrm{cm}^{2}$ calculated from the finite element analysis.

Of interest is that our recent work also indicated that a single dose of $25 \mathrm{~mW} / \mathrm{cm}^{2}$ stimulated subsequent in vitro differentiation and mineralization [28]. These observations suggest that a single application of ultrasound could trigger cellular responses leading to proliferation and differentiation of odontoblasts. Further research is warranted to elucidate the clinical potential and biophysics of ultrasound within the dental pulp in order to harness and develop a suitable and efficient therapeutic tool for tooth repair.

\section{Conclusions}

This study has modelled the transmission of low-intensity low-frequency ultrasound through the outer mineralized layers of the teeth to the dental pulp chamber. In addition, this research demonstrated that a single treatment of lowfrequency ultrasound stimulates dental pulp cell proliferation using long-term MDPC-23 cell cultures. Thus, the data provide evidence that exposure of the teeth to lowfrequency ultrasound may generate a therapeutic intensity within the dental pulp that may facilitate new reparative dentine formation.

\section{Additional files}

Additional file 1: Snapshots of pressure and intensity plots of ( $30 \mathrm{kHz}, 70 \mathrm{~Pa}$ ) ultrasonic wave throughout the tooth.

Additional file 2: Snapshots of pressure and intensity plots of $(45 \mathrm{kHz}, 31 \mathrm{kPa}$ ) ultrasonic wave throughout the tooth.

Additional file 3: Snapshots of pressure and intensity plots of $(500 \mathrm{kHz}, 200 \mathrm{kPa})$ ultrasonic wave throughout the tooth.

Additional file 4: Snapshots of pressure and intensity plots of $(1 \mathrm{MHz}, 19 \mathrm{kPa}$ ) ultrasonic wave throughout the tooth.

\section{Competing interests}

The authors declare that they have no competing interests.

\section{Authors' contributions}

SRG mentored the finite element modelling portion of this research project, provided guidelines and protocols for all simulation studies, analysis and interpretation of the data, wrote half of the manuscript and was responsible for the compilation of the complete manuscript and final submission. UP carried out the laboratory measurements and interpretation of the data. ADW contributed his expertise in ultrasound-based techniques as applied to dentistry. BAS mentored the in vitro study portion of this research project, provided clinical information about this application, participated in the interpretation of the data and wrote the sections pertaining to this aspect of the manuscript. All authors read and approved the final manuscript. 


\section{Acknowledgements}

The finite element portion of this work was performed within the framework of the Capstone Senior Design component in the School of Engineering and Applied Sciences, Ultrasound Research Laboratory at Hofstra University. A special thank you goes to Mr. Sabir Liakat, Mr. Dan Apgar, Mr. Dan Audette and Mr. Andre Blackwood for developing the initial AutoCAD models for the teeth, setting up the COMSOL program and collecting the data while working on their senior design.

\section{Author details}

'School of Engineering and Applied Sciences, Ultrasound Research Laboratory, Hofstra University, 104 Weed Hall, Hempstead, NY 11549, USA. ${ }^{2}$ Orthopaedics Research Laboratory, FIMR, North Shore Hospital, Manhassett, NY 11030, USA. ${ }^{3}$ School of Dentistry, College of Medical and Dental Sciences, University of Birmingham, St Chad's Queensway, Birmingham B4 6NN, UK.

Received: 16 April 2013 Accepted: 1 June 2013

Published: 1 August 2013

\section{References}

1. Walmsley AD. Applications of ultrasound in dentistry. Ultrasound Med Biol. 1988: 14:7-14

2. Ghorayeb SR, Valle T. Experimental evaluation of human teeth using noninvasive ultrasound: echodentography. IEEE Trans Ultrason Ferroelec Freq Contr. 2002; 49(10):1437-43.

3. Ghorayeb SR, Bertoncini CA, Hinders MK. Ultrasonography in dentistry. IEEE Trans Ultrason Ferroelec Freq Contr. 2008; 55(6):1256-66.

4. Dalecki D. Mechanical bioeffects of ultrasound. Annu Rev Biomed Eng. 2004; 6:229-48.

5. Ter Haar G. Therapeutic applications of ultrasound. Prog Biophys Molec Biol. 2007; 93:111-29.

6. Claes $L$, Willie $B$. The enhancement of bone regeneration by ultrasound. Prog Biophys Mol Biol. 2007; 93:384-98.

7. Petersen PE. The World Oral Health Report 2003: continuous improvement of oral health in the 21st century - the approach of the WHO Global Oral Health Programme. Community Dentistry and Oral Epidemiology. 2003: 32(1):3-24.

8. Lucarotti PS, Holder RL, Burke FJ. Outcome of direct restorations placed within the general dental services in England and Wales (Part 1): variation by type of restoration and re-intervention. J Dent. 2005; 33:805-15.

9. Smith $\mathrm{AJ}$, Lesot $\mathrm{H}$. Induction and regulation of crown dentinogenesis: embryonic events as a template for dental tissue repair? Crit Rev Oral Biol Med. 2001; 12:425-37.

10. Scheven BA, Shelton RM, Cooper PR, Walmsley AD, Smith AJ. Therapeutic ultrasound for dental tissue repair. Med Hypoth. 2009; 73:591-93.

11. Reher P, Elbeshir ENI, Harvey W, Meghii S, Harris M. The stimulation of bone formation in vitro by therapeutic ultrasound. Ultrasound Med Biol. 1997; 23:1251-58.

12. Harle J, Mayia F, Olsen I, Salih V. Effects of ultrasound on transforming growth factor-beta genes in bone cells. Eur Cell Mater. 2005; 10:70-6.

13. Khan $Y$, Laurencin CT. Fracture repair with ultrasound: clinical and cellbased evaluation. J Bone Joint Surg Am. 2008; 90(1):138-44.

14. Scheven BA, Millard JL, Cooper PR, Lea SC, Walmsley AD, Smith AJ. Short-term in vitro effects of low frequency ultrasound on odontoblast-like cells. Ultrasound Med Biol. 2007; 33:1475-82.

15. Scheven BA, Man J, Millard JL. VEGF and odontoblast-like cells: stimulation by low frequency ultrasound. Arch Oral Biol. 2009; 54(2):185-91.

16. Ghorayeb SR, Xue T, Lord W. A finite element study of ultrasonic wave propagation in a tooth phantom. J Dent Res. 1998; 77(1):39-49.

17. Ghorayeb SR, Maione E. Diagnostic ultrasound for the imaging of teeth: a comparison between experimental results and simulation models. IEEE Ultrasonics Symp. 2000; 2000:1387-90

18. Ghorayeb SR, Maione E, La Magna V. Modeling of ultrasonic wave propagation in teeth using PSpice: a comparison with finite element models. IEEE Trans Ultrason, Ferroelec, Frea Contr. 2001; 48(4):1124-31.

19. Ghorayeb SR, Xue T, Lord W. Ultrasonic imaging of teeth for early detection of abscesses. IEEE International Ultrasonics Symp. 1997; 1997:5-8.

20. Dodd SP, Cunningham JL, Miles AW, Gheduzzi S, Humphrey VF. Ultrasonic propagation in cortical bone mimics. Phys Med Biol. 2006; 51:4635-47.
21. Kaufman JJ, Luo G, Siffert RS. Ultrasound simulation in bone. IEEE Trans Ultrason Ferroelec Frea Cont. 2008; 55:1205-18.

22. Rudd $K$, Hinders M. Simulation of incident nonlinear sound beam and $3 D$ scattering from complex targets. Journ Comp Acoust. 2008; 16(3):427-45.

23. Rudd K, Bertoncini C, Hinders M. Simulations of ultrasonographic periodontal probe using the finite integration technique. Open Acoust J. 2008; 1:1-19.

24. Olgart L, Edwall L, Gazelius B. Involvement of afferent nerves in pulpal blood-flow reactions in response to clinical and experimental procedures in the cat. Arch Oral Biol. 1991; 36:575-81.

25. El-Bialy T. Low intensity pulsed ultrasound: a laboratory and clinical

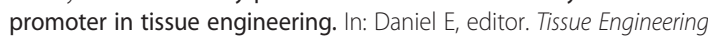
Volume 15. 1st ed. IN-TECH: Croatia; 2010: p. 307-22.

26. Frost HM. Bone "mass" and the "mechanostat": a proposal. Anat Rec. 1987; 219:1-9.

27. Skerry TM. One mechanostat or many? Modifications of the site-specific response of bone to mechanical loading by nature and nurture. J Musculoskelet Neuronal Interact. 2006; 6:122-27.

28. Man J, Shelton RM, Cooper PR, Scheven BAA. Low-intensity low-frequency ultrasound promotes proliferation and differentiation of odontoblast-like cells. J Endod. 2012; 38:608-13.

doi:10.1186/2050-5736-1-12

Cite this article as: Ghorayeb et al:: Biophysical characterization of lowfrequency ultrasound interaction with dental pulp stem cells. Journal of Therapeutic Ultrasound 2013 1:12.

\section{Submit your next manuscript to BioMed Central and take full advantage of:}

- Convenient online submission

- Thorough peer review

- No space constraints or color figure charges

- Immediate publication on acceptance

- Inclusion in PubMed, CAS, Scopus and Google Scholar

- Research which is freely available for redistribution 